\title{
The phase diagram of the multi-dimensional Anderson localization via analytic determination of Lyapunov exponents
}

\author{
V.N. Kuzovkov ${ }^{1}$ and W. von Niessen ${ }^{2}$ \\ ${ }^{1}$ Institute of Solid State Physics, University of Latvia, 8 Kengaraga Street, LV - 1063 RIGA, Latvid* \\ ${ }^{2}$ Institut für Physikalische und Theoretische Chemie, Technische Universität Braunschweig, \\ Hans-Sommer-Straße 10, 38106 Braunschweig, Germany
}

(Dated: Received November 21, 2018)

\begin{abstract}
The method proposed by the present authors to deal analytically with the problem of Anderson localization via disorder [J.Phys.: Condens. Matter 14 (2002) 13777] is generalized for higher spatial dimensions D. In this way the generalized Lyapunov exponents for diagonal correlators of the wave function, $\left\langle\psi_{n, \mathbf{m}}^{2}\right\rangle$, can be calculated analytically and exactly. This permits to determine the phase diagram of the system. For all dimensions $D>2$ one finds intervals in the energy and the disorder where extended and localized states coexist: the metal-insulator transition should thus be interpreted as a first-order transition. The qualitative differences permit to group the systems into two classes: low-dimensional systems $(2 \leq D \leq 3)$, where localized states are always exponentially localized and high-dimensional systems $\left(D \geq D_{c}=4\right)$, where states with non-exponential localization are also formed. The value of the upper critical dimension is found to be $D_{0}=6$ for the Anderson localization problem; this value is also characteristic of a related problem - percolation.
\end{abstract}

PACS numbers: $72.15 . \mathrm{Rn}, 71.30 .+\mathrm{h}$

\section{INTRODUCTION}

\section{A. Experiment and theory}

Disorder leads to important physical effects which are of quantum mechanical origin. This has been revealed by Anderson [1] in the study of a disordered tight-binding model. This problem has attracted great attention over many decades. A breakthrough came with the scaling theory of localization [2]. All states in a one-dimensional system (1-D) are localized, whereas in 3-D a metal-insulator transition occurs. An analytic solution is only known for the 1-D problem [3]. Although there was no general analytical solution available, there was consensus that in 2-D all states are localized.

For quite some time after the advent of the scaling theory, many believed it to be essentially under control. This view is less secure nowadays, in part because recent experiments have challenged conventional wisdom about disordered 2-D systems. The 2-D case still presents a problem which has become apparent by experiments [4, 5, 6, 7]. These experiments are still being discussed controversially. Experimental reality is certainly more complex than a simple tight-binding model, but these results provide a good reason for revisiting this classic theoretical problem.

Recently we have been able to solve the 2-D case analytically [8]. We have shown that in principle there is the possibility that the phase of delocalized states exists for a non-interacting electron system. For energies and disorder, where extended states may exist we find a coexistence of these localized and extended states. Thus the Anderson metal-insulator transition exists and should be regarded as a first order phase transition. Consequently we have returned to the old idea of Mott 9, 10 that the metal-insulator transition is discontinuous. This alternative idea was in its history completely abandoned with the advent of the scaling theory of localization. Thus we expect a renaissance of it.

This result implies the failure of the scaling theory of Anderson localization. Although this paper is published [8] and constitutes the basis for a new analytical investigation of the Anderson problem in the present paper for the case of higher dimensions (N-D problem) one has to accept the following: (i) the paper [8] requires an independent confirmation, which requires a certain time; (ii) in the history of the problem one has developed quite a few conceptions and this leads to a critical attitude towards the new theory - i.e. there is a resistance - as it departs from conventional wisdom. This asks for a critical evaluation of the new theory and its results [1, 12]. One should acknowledge that the problem is many-sided and quite complex. Thus logical errors are possible which do not lie at the surface. Perhaps a reference to B. Pascal is appropriate here: A truth is so delicate that any small deviation from it leads you to a

*Electronic address: kuzovkov@latnet.lv 
mistake, but this mistake is also so delicate that after a small retreat you find yourselves in a truth again. Without an exact analytic solution a discussion cannot lead to firm results.

Let us add at this point some reflections of a historical nature. It is well known that exact results in the field of phase transitions are exceedingly rare (so to say they are exceptions) and they exist only under certain conditions and limitations. The situation in the field of Anderson localization shows in this respect a certain similarity to the history of the theory of the second order phase transitions and of the Ising model. The phenomenological theoretical work by Landau [13] and the numerical work by Bethe [14] were prior to Onsager's analytical theory [15] and they were well accepted. But the results of the analytical theory departed strongly from the phenomenological and numerical results in that it gave different critical exponents. But there are limitations to the analytical theory. The exact solution of Onsager for the Ising model is only possible for 2-D, without magnetic field and only for the case of an interaction between nearest neighbours. This is also the case in our present investigation. An exact solution is only possible (a) for the tight-binding approximation with (b) diagonal disorder, where (c) on-site potentials are independently and identically distributed, i.e. for the conventional Anderson model.

A contradiction between results from phenomenological theories (Landau, scaling theory) and analytical results (Onsager, the present work) have (Onsager) or might (the present case) lead to scientific advances. The metalinsulator transition is a phase transition. In the case of such phase-transitions the results tend to be unstable and depend on the approximations. An exact solution thus rarely shows an agreement with results from a phenomenological theory and numerical work, because the two latter methods make use of additional approximations and assumptions, whose consequences are hardly controllable. To return to the advances made possible by Onsager's analytical theory: V. Ginzburg got in the year 2003 the nobel prize for the work (together with L. Landau [16]), in which he used the phenomenological theory of second order phase transitions. This all means simply that phenomenological and numerical results frequently require certain corrections, which is only then possible, when an exact solution is known.

The main aim of the present article is thus the generalization of the mathematical tools of the previous article [8] to the case of higher dimensional spaces and a physical interpretation of the new results.

\section{B. Structure of the present article}

The outline of the present article is a follows. The article [8] opens the possibility to determine the phase diagram of the multi-dimensional Anderson localization analytically. This is based on the analytic calculation of Lyapunov exponents. The argumentation is given at the beginning of chapter 2 in detail. There we also give a short derivation of the equations for the N-D Anderson localization problem, and a necessary summary of the results of the article [8]. The connection between the Anderson localization problem and signal theory is discussed and the most important concept of the proposed method - the filter $H(z)$ - is defined. To understand fully these aspects of the theory a knowledge of the first paper [8] is recommended. The filter $H(z)$ is generalized for higher dimensions $\mathrm{D}$ of the space. The investigation of its properties and the corresponding physical interpretation constitute the content of several chapters. The theory for high-dimensional systems $(D \geq 4)$ is presented in chapter 3, whereas the theory for lowdimensional systems is given in chapter 4 . An appendix deals with the mathematical conditions for the physical interpretation of the filter $H(z)$ and a more detailed discussion of related aspects of the problem. Because of the length of the present article we omit both the mathematical details of the theory as well as an extended discussion of the implications of the results. This will be given in a future publication, where also a different procedure for part of the derivation is used instead of an average over initial conditions as done here.

\section{NEW METHODS}

\section{A. Anderson localization and generalized diffusion}

Recently we have been able to solve the 2-D case analytically [8]. The tight-binding equation in 2-D is solved for the wave function $\psi_{n, m}$ and the second moments (diagonal correlators $\left\langle\psi_{n, m}^{2}\right\rangle$ ):

$$
\psi_{n+1, m}=\left(E-\varepsilon_{n, m}\right) \psi_{n, m}-\psi_{n-1, m}-\sum_{m^{\prime}} \psi_{n, m+m^{\prime}},
$$

where the summation over $m^{\prime}$ runs over the nearest neighbours of site $(n, m)$ in layer $n$ that is in a space of dimension $p=D-1=1$. We assume taking the limit to infinite size $L \rightarrow \infty$ in $p$-space. The equation is solved with an initial condition

$$
\psi_{0, m}=0, \psi_{1, m}=\alpha_{m} .
$$


The on-site potentials $\varepsilon_{n, m}$ are independently and identically distributed with existing first two moments, $\left\langle\varepsilon_{n, m}\right\rangle=0$ and $\left\langle\varepsilon_{n, m}^{2}\right\rangle=\sigma^{2}$.

The mathematical idea shortly discussed above is based on a simple physical idea. The aim of the article [8] consisted in determining a phase diagram for the Anderson localization problem. To this purpose the concept of the Lyapunov exponent $\gamma[\underline{8}]$ is extremely well suited. The Lyapunov exponent plays the role of an order parameter: $\gamma \equiv 0$ for the metallic phase and $\gamma \neq 0$ for the insulating phase. The value $\gamma \neq 0$ for the insulating phase implies a divergence of certain mean values as a function of the index $n$. If we understand what this divergence means it will become clear, which quantities have to be calculated.

This divergence is a general property of a number of different stochastic systems. To give a clear picture we consider in the following the equations for 1-D systems and in particular for diffusion (random walks)

$$
\psi_{n+1}=\psi_{n}+\varepsilon_{n}
$$

and Anderson localization

$$
\psi_{n+1}=\left(E-\varepsilon_{n}\right) \psi_{n}-\psi_{n-1} .
$$

For symmetric diffusion, $\left\langle\varepsilon_{n}\right\rangle=0,\left\langle\varepsilon_{n}^{2}\right\rangle=\sigma^{2}$, the simplest and most important characteristic is the second moment $\left\langle\psi_{n}^{2}\right\rangle=\psi_{0}^{2}+\sigma^{2} n$. A divergence of this variable for $n \rightarrow \infty$ is an unambiguous proof for the existence of diffusion. It is also analytically possible to calculate other even moments, e.g. $\left\langle\psi_{n}^{4}\right\rangle$. These, however, do not supply new information. All even moments diverge simultaneously, and they do this as a power of $n$. A similar divergence also occurs for other mean values of the functions of $\left|\psi_{n}\right|$, e.g. $\left\langle\left|\psi_{n}\right|\right\rangle$; however, these can only be calculated numerically. In other words, for the determination of the existence of diffusion it is fully sufficient to calculate only one moment, the second moment.

The equation (4) is nothing else but a generalization of equation (3). The mean value $\left\langle\psi_{n}^{2}\right\rangle=f(n)$ describes in this case generalized diffusion. If $f(n)$ is bounded, $f(n)<\infty$, then the proper dynamics in equation (44) is stable and we have no diffusion (Lyapunov exponent $\gamma \equiv 0$ ). This corresponds in a physical interpretation to the existence of delocalized states. A divergence of the function $f(n)$ for $n \rightarrow \infty$ corresponds to generalized diffusion (localized states); in this case one could distinguish in addition between non-exponential localization $(f(n)$ is a non-exponential function) and exponential localization $(f(n) \propto \exp (2 \gamma n)$ with $\gamma \neq 0)$.

It is quite important to stress here that the formation of diffusion in a dynamic system represents always a type of phase transition. Therefore one can easily find the corresponding critical values and determine the phase diagram of the system. As for proper diffusion, an analytic calculation for eq. (4) is only possible for even moments [3, 8].

For these reasons it is fully sufficient to consider only the equations for the second moments (correlators) to determine the phase diagram of the system [8]. This idea has a certain similarity to the method of Pendry [17]. Pendry has introduced the density matrix to the problem of waves in disordered systems. However, he was of the opinion (without giving a proof), that for Anderson localization all higher-order density matrices and hence all higher moments need to be considered. This program is practically unfeasible, so Pendry limited himself in [17] to the second moment. Instead of solving the equations for the second moments exactly Pendry has mainly studied the properties of the transfer matrix and the process of its diagonalization. For the transfer matrix, however, the investigation of the thermodynamic limit is impossible (see discussion in [12]), and consequently in this way the phase diagram of the system cannot be obtained.

\section{B. Equations for correlators}

In the present paper we present the analytic solution for the general case $D>2$. The knowledge of paper [8] is prerequisite for understanding the present one as it contains the full formalism. The generalization of this formalism to the N-D case presents no problem (see below). The main eqs. (10),(2) remain valid, only scalar quantities become vector quantities.

The tight-binding equation in $1+p$ dimension is (primed indices are summed)

$$
\begin{gathered}
\psi_{n+1, \mathbf{m}}=-\varepsilon_{n, \mathbf{m}} \psi_{n, \mathbf{m}}-\psi_{n-1, \mathbf{m}}+\mathcal{L}_{\mathbf{m}, \mathbf{m}^{\prime}} \psi_{n, \mathbf{m}^{\prime}}, \\
\mathcal{L}_{\mathbf{m}, \mathbf{m}^{\prime}}=E \delta_{\mathbf{m}, \mathbf{m}^{\prime}}-\sum_{\mathbf{m}^{\prime \prime}} \delta_{\mathbf{m}+\mathbf{m}^{\prime \prime}, \mathbf{m}^{\prime}}
\end{gathered}
$$

(summation over $\mathbf{m}^{\prime \prime}$ runs over the nearest neighbours) with initial condition $\psi_{0, \mathbf{m}}=0$ and $\psi_{1, \mathbf{m}}=\alpha_{\mathbf{m}}$. 
One introduces the correlators (the averages are taken over disorder)

$$
\begin{gathered}
x(n)_{\mathbf{m}, \mathbf{l}}=\left\langle\psi_{n, \mathbf{m}} \psi_{n, \mathbf{l}}\right\rangle, \\
y(n)_{\mathbf{m}, \mathbf{l}}=\left\langle\psi_{n, \mathbf{m}} \psi_{n-1, \mathbf{l}}\right\rangle .
\end{gathered}
$$

From the eq.(5) one easily obtains the system of equations:

$$
\begin{array}{r}
x(n+1)_{\mathbf{m}, \mathbf{l}}=\delta_{\mathbf{m}, \mathbf{l}} \sigma^{2} x(n)_{\mathbf{m}, \mathbf{l}}+x(n-1)_{\mathbf{m}, \mathbf{l}}+ \\
\mathcal{L}_{\mathbf{m}, \mathbf{m}^{\prime}} x(n)_{\mathbf{m}^{\prime}, \mathbf{l}^{\prime}} \mathcal{L}_{\mathbf{l}^{\prime}, \mathbf{l}}-\mathcal{L}_{\mathbf{m}, \mathbf{m}^{\prime}} y(n)_{\mathbf{m}^{\prime}, \mathbf{l}}-\mathcal{L}_{\mathbf{l}, \mathbf{l}^{\prime}} y(n)_{\mathbf{l}^{\prime}, \mathbf{m}} \\
y(n+1)_{\mathbf{m}, \mathbf{l}}=-y(n)_{\mathbf{l}, \mathbf{m}}+\mathcal{L}_{\mathbf{m}, \mathbf{m}^{\prime}} x(n)_{\mathbf{m}^{\prime}, \mathbf{l}}
\end{array}
$$

They can be solved explicitly by introducing of the Z-transform 18

$$
\begin{aligned}
& X(z)_{\mathbf{m}, \mathbf{1}}=\sum_{n=0}^{\infty} \frac{x(n)_{\mathbf{m}, \mathbf{1}}}{z^{n}} \\
& Y(z)_{\mathbf{m}, \mathbf{1}}=\sum_{n=0}^{\infty} \frac{y(n)_{\mathbf{m}, \mathbf{l}}}{z^{n}}
\end{aligned}
$$

which turn the equations into

$$
\begin{array}{r}
\left(z-z^{-1}-\sigma^{2} \delta_{\mathbf{m}, \mathbf{l}}\right) X(z)_{\mathbf{m}, \mathbf{l}}-x(1)_{\mathbf{m}, \mathbf{l}}=\mathcal{L}_{\mathbf{m}, \mathbf{m}^{\prime}} X(z)_{\mathbf{m}^{\prime}, \mathbf{l}^{\prime}} \mathcal{L}_{\mathbf{l}^{\prime}, \mathbf{l}} \\
-\mathcal{L}_{\mathbf{m}, \mathbf{m}^{\prime}} Y(z)_{\mathbf{m}^{\prime}, \mathbf{l}}-\mathcal{L}_{\mathbf{l}, \mathbf{l}^{\prime}} Y(z)_{\mathbf{l}^{\prime}, \mathbf{m}}, \\
z Y(z)_{\mathbf{m}, \mathbf{l}}=-Y(z)_{\mathbf{l}, \mathbf{m}}+\mathcal{L}_{\mathbf{m}, \mathbf{m}^{\prime}} X(z)_{\mathbf{m}^{\prime}, \mathbf{l}}
\end{array}
$$

Iteration of the second equation yields

$$
Y(z)_{\mathbf{m}, \mathbf{l}}=\frac{z}{z^{2}-1} \mathcal{L}_{\mathbf{m}, \mathbf{m}^{\prime}} X(z)_{\mathbf{m}^{\prime}, \mathbf{l}}-\frac{1}{z^{2}-1} X(z)_{\mathbf{m}, \mathbf{l}^{\prime}} \mathcal{L}_{\mathbf{l}^{\prime}, \mathbf{l}}
$$

We then obtain an equation for $X(z)_{\mathbf{m}, \mathbf{l}}$ only, which can be solved by double Fourier expansion. A complete presentation of the method is rather lengthy and will thus be the subject of a following paper. An shortcut which quickly leads to the correct equations is suggested in section 3.2 of [8] ): averaging over translations in $p$-space of boundary conditions, $\alpha_{\mathbf{m}}$.

Upon averaging over an ensemble of initial conditions (as described in [8]) such that $\overline{\alpha_{\mathbf{m}} \alpha_{\mathbf{m}^{\prime}}}=\Gamma_{\mathbf{m}-\mathbf{m}^{\prime}}$, the problem is translation-invariant in transverse directions. We then put:

$$
X(z)_{\mathbf{m}, \mathbf{l}}=\int \frac{d^{p} \mathbf{k}}{(2 \pi)^{p}} X(z, \mathbf{k}) e^{i \mathbf{k}(\mathbf{m}-\mathbf{l})} .
$$

After averaging over initial conditions the diagonal correlator becomes independent of $\mathbf{m}$ :

$$
\begin{array}{r}
x(n)_{\mathbf{m}, \mathbf{m}} \equiv x_{n}, \\
X(z)_{\mathbf{m}, \mathbf{m}} \equiv X(z)=\int \frac{d^{p} \mathbf{k}}{(2 \pi)^{p}} X(z, \mathbf{k}) .
\end{array}
$$

We obtain the final equations

$$
\begin{array}{r}
\frac{(z-1)}{(z+1)}\left[w^{2}-\mathcal{E}^{2}(\mathbf{k})\right] X(z, \mathbf{k})=\Gamma(\mathbf{k})+\sigma^{2} X(z), \\
\mathcal{E}(\mathbf{k})=E-2 \sum_{j=1}^{p} \cos \left(k_{j}\right), \\
w^{2}=\frac{(z+1)^{2}}{z},
\end{array}
$$

or

$$
\begin{array}{r}
X(z)=H(z) X^{(0)}(z), \\
X^{(0)}(z)=\frac{(z+1)}{(z-1)} \int \frac{d^{p} \mathbf{k}}{(2 \pi)^{p}} \frac{\Gamma(\mathbf{k})}{\left[w^{2}-\mathcal{E}^{2}(\mathbf{k})\right]}, \\
\frac{1}{H(z)}=1-\sigma^{2} \frac{(z+1)}{(z-1)} \int \frac{d^{p} \mathbf{k}}{(2 \pi)^{p}} \frac{1}{\left[w^{2}-\mathcal{E}^{2}(\mathbf{k})\right]}
\end{array}
$$


where the $X^{(0)}(z)$ (or $\left.x_{n}^{(0)}\right)$ refer to the ordered system $(\sigma \equiv 0)$ and the $X(z)$ (or $\left.x_{n}\right)$ to the disordered one $(\sigma \neq 0$ ). For eq. 222) the inverse Z-transform gives the convolution property [18]:

$$
x_{n}=\sum_{l=0}^{n} x_{l}^{(0)} h_{n-l}
$$

with

$$
H(z)=\sum_{n=0}^{\infty} \frac{h_{n}}{z^{n}}
$$

\section{Anderson localization and signal theory}

The essential point in the analysis with respect to the localized or extended character of the states is to make use of signal theory 18] from electrical engineering and switch from an investigation of the moments $x_{n}$ to an analysis of the filter functions $h_{n}$.

In the theory of signals [18], $x_{n}^{(0)}$ (or $X^{(0)}(z)$ ) is the input signal, and $x_{n}$ (or $X(z)$ ) is the output. Asymptotic behaviour of the solution is completely determined by the filter $h_{n}$ (or $H(z)$ ). The concept of the system function is a general and abstract description of the problem of localization. Thus the filter function has to be analysed to obtain general results and not the multitude of signals. This has been done in [8] for the 2-D case. This procedure has a certain similarity with the transition to an operator formalism in quantum mechanics. Particular signals $x_{n}$ depend on the initial conditions used and do not carry much physical information because of the unconventional normalization [8]. For the localization problem the only property that matters is whether a signal belongs to the bounded or unbounded class and this can be derived from the filter.

The essence of localization is contained in the filter $H(z)$. We study the filter $H(z)$ with properties described by generalized Lyapunov exponents [8, 19]. The filter is a fundamental function of the disorder $\sigma$ only.

A filter $h_{n}$ is uniquely characterized by a pole-zero diagram of its image $H(z)$ which is a plot of the locations of the poles $\lambda_{i}$ and zeros of $H(z)$ in the complex- $z$ plane. We provide just a brief summary here, for more details consult [8, 18]. The signals $x_{n}^{(0)}$ and $x_{n}$ are real, therefore $H(z)$ will have poles and zeros that are either on the real axis, or come in conjugate pairs. For the inverse $Z$-transform $H(z) \Rightarrow h_{n}$ one needs to know the region of convergence (ROC). Physical considerations dictate that only causal filters $\left(h_{n}=0\right.$ for $\left.n<0\right)$ should be considered. They have ROCs outside a circle that intersects the pole with $\max \left|\lambda_{i}\right|$. A causal filter is stable (bounded input yields a bounded output) if the unit circle $|z|=1$ is in the ROC. Note that the explicit calculation of $h_{n}$ by the inverse $Z$-transform is not necessary, and it is also not feasible analytically due to the complexity of the function $H(z)$. Only the type of the filter - stable or unstable - needs to be determined. The delocalized states (bounded output) are obtained by transforming the physical solutions inside the band (bounded input) provided that the filter $H(z)$ is stable. Seeking for poles is quite a simple analytical task which gives rather general results by elementary methods.

As an example for the general and abstract description of the problem of localization as stated above let us consider the following problem. It is well-known that disorders broadens the band; new states outside the old band arise for $|E|>E_{b}=2 D$. Are among these new states also extended states? Numerical work for $D=3$ [20, 21] ascertains this. This is the socalled reentrant behaviour of the mobility edge: the change from localized to extended states and back to localized ones upon increasing the disorder occurs for certain fixed energies. Because in the literature there is no physical explanation for this phenomenon one has simply accepted these results without critically examining them.

With the help of signal theory we have found a particular transformation, which gives a connection between the states in an ideal system (zero disorder) and states in a system with disorder. Extended states (zero disorder) as input (bounded) signal transform into localized states (nonzero disorder) as output (unbounded) signal if the filter which is responsible for this transformation is unstable. If the filter is stable, then extended states (bounded input signal) transform into extended states (bounded output signal). It is known that for zero disorder outside the band, $|E|>E_{b}$, there do exist only mathematical solutions which cannot be normalized. These correspond to an unbounded input signal. It is impossible to find a filter which permits a transformation of the unbounded input signal (mathematical solution) into a bounded output signal (extended states in $|E|>E_{b}$ ). The reverse - the transformation of an unbounded input signal (mathematical solution) into a unbounded output signal (localized states in $|E|>E_{b}$ ) - is on the other hand possible. I.e. the mathematical procedure developed by us generates in this case a negative answer to the posed question.

We have shown in [8] that the filter $H(z)$ is a non-analytic function of the complex variable $z$; this result remains valid also in the multi-dimensional case. The unit circle $|z|=1$ divides the complex plane into two analytic domains: 
the interior and exterior of the unit circle. The inverse Z-transform is quite generally defined via contour integrals in the complex plane

$$
h_{n}=\frac{1}{2 \pi i} \oint H(z) z^{n} \frac{d z}{z} .
$$

and this definition is only possible in an analytic domain. In this way in the formal analysis of the problem multiple solutions result. The first solution $H_{+}(z)$ is defined outside the unit circle and always exists. The filter $H_{+}(z)$ describes localized states and it is possible to connect its properties with the notion of the localization length [8]. The second solution $H_{-}(z)$ is defined inside the unit circle and does not always represent a solution which can be physically interpreted (this is the mathematical consequence that the filter be causal). The filter $H_{-}(z)$ describes delocalized states. The coexistence of the two solutions was physically interpreted in [8, 12] as the coexistence of two phases - an insulating and a metallic one. Then the metal-insulator transition should be looked at from the basis of first-order phase transition theory.

\section{Conformal mapping}

The $p$-dimensional integral on the r.h.s. of eq. (24) can be reduced to a one-dimensional integral. Consider the identity

$$
\frac{1}{w^{2}-\mathcal{E}^{2}(\mathbf{k})}=\int_{-\infty}^{\infty} \frac{\delta\left(y+\sum_{j=1}^{p} 2 \cos \left(k_{j}\right)\right) d y}{w^{2}-(E+y)^{2}} .
$$

The integral representation of the Dirac $\delta$-function and the Bessel function

$$
J_{0}(x)=\frac{1}{2 \pi} \int_{-\pi}^{\pi} e^{i x \cos (k)} d k
$$

will be used. Following [8], let us define a complex parameter $w=u+i v$ from eq. (21) in the upper half-plane, $v \geq 0$. Using the methods of complex variable theory we get

$$
\frac{1}{(2 \pi)^{p}} \int \frac{d \mathbf{k}}{w^{2}-\mathcal{E}^{2}(\mathbf{k})}=\frac{1}{i w} Y_{D}(w, E),
$$

where

$$
Y_{D}(w, E)=\int_{0}^{\infty}\left[J_{0}(2 t)\right]^{D-1} \cos (E t) \exp (i w t) d t .
$$

Changing the complex variable $z$ to the parameter $w$ corresponds to the conformal mapping of the inner part $[|z| \leq 1$, $\left.\left.w=-\left(z^{1 / 2}+z^{-1 / 2}\right)\right)\right]$ or the outer part $\left[|z| \geq 1, w=\left(z^{1 / 2}+z^{-1 / 2}\right)\right]$ of the circle onto the upper half-plane. The circle itself maps onto the interval $[-2,2]$. Note also that if $H(z)=0$ has complex conjugate poles, then on the upper $w$ half-plane they differ only by the sign of $u$. To avoid complicated notations, we seek for poles in the sector $u \geq 0, v \geq 0$ and double their number if we find any.

The inverse function [8]

$$
z=-1+\frac{w^{2}}{2} \pm \frac{w}{2} \sqrt{w^{2}-4}
$$

is double-valued. Its branch with the minus sign maps the $w$ sector onto the inner part of the half-circle $(|z| \leq 1)$. The second branch with the plus sign gives a mapping onto the half-plane with the half-circle excluded $(|z| \geq 1)$. Therefore in the parametric $w$-representation

$$
\frac{z+1}{z-1}= \pm \frac{w}{\sqrt{w^{2}-4}}
$$

and

$$
\frac{1}{H_{ \pm}(z)}=1 \pm \sigma^{2} i \frac{Y_{D}(w, E)}{\sqrt{w^{2}-4}}
$$




\section{HIGH-DIMENSIONAL SYSTEMS}

\section{A. Upper critical dimension}

It is generally assumed that 2-D systems mark the borderline between high and low dimension 22. The existence of a transition in 3-D is not questioned (high-dimensional systems). These assumptions, however, originate from the scaling theory of localization. Here the marginal dimension is $D_{M}=2$, and a phase transition exists only for $D>D_{M}$. Thus perturbation theory 4] for $D=2+\varepsilon(\varepsilon \ll 1)$ is possible. The effect of statistical fluctuations cause a change of regime at $D_{c}=4[10,23]$; in this way the upper critical dimension for localization $D_{c}$ arises. For $D>D_{c}$ there should not exist a phase transition.

On the one hand these statements referring to higher dimensions are numerically nearly impossible to ascertain 24]. Statistics is bad and the length of the system $L$ very small. Even for $D=3$ progress towards extracting reliable numerical estimates of critical quantities has been remarkably difficult 25]. On the other hand, if the results from the scaling theory of localization for 2-D systems are faulty (this is what we claim), then the corresponding division of the systems into low and high dimensional ones is also wrong. Here one must develop an alternative picture.

In the theory of critical phenomena [26, 27] many systems belong to a class, where an upper critical dimension $D_{0}$ has a totally different physical meaning. It denotes the dimension, from where on the mean field approximation is exact, or where with other words all critical exponents reach stationary values. I.e. in this case the phase transition does exist also for $D>D_{0}$; only in the limit $D \rightarrow \infty$ the transition disappears, simply because the corresponding critical values have gone to infinity. Systems of quite different physical nature may have the same value of the upper critical dimension $D_{0}$. E.g. one finds $D_{0}=4$ not only for the theory of magnetism [26, 27] (in this case there exists also the marginal dimension, below which no phase transition is possible), but also in kinetics (cooperative phenomena in bimolecular processes by diffusion-controlled reactions) [28, 29]; in the latter case, however, there is no marginal dimension. Another example is percolation, where the upper critical dimension is $D_{0}=6$ [30].

\section{B. Stability and poles: solution $H_{+}(z)$}

Let us start first from a purely mathematical comment: the integral $Y_{D}(w, E)$ is always finite for all $w=u+i v$ in the sector $u \geq 0, v \geq 0$ for high-dimensional systems with $D \geq 4$. This fact clearly follows from the asymptotic behaviour of the Bessel function for large values of its argument, $\bar{J}_{0}(2 t) \approx \frac{1}{\sqrt{\pi t}} \cos (2 t-\pi / 4)$. We shall further on see that the dimensionality $D=D_{c}=4$ is critical for localization, although this is no proper upper critical dimension $D=D_{0}$, which we shall determine below. Let us consider therefore the properties of the filter-functions in this region of the value of the dimension of space.

Let us consider first the solution $H_{+}(z)$. According to [18], the ROC of the causal filter is defined by the inequality $|z|>\max \left|\lambda_{i}\right|$, where $\lambda_{i}$ are the poles. The case when the system function has a pole at $z=\lambda>1$ is the simplest one for an interpretation. In terms of signal theory 18 the filter $H_{+}(z)$ is unstable since the pole lies outside the unit circle $|z|=1$ in the complex $z$-plane. As shown in [8], such a filter describes exponentially localized states (insulating phase). In order to see this one can exploit a basic inverse Z-transform [18]:

$$
H(z)=z /(z-\lambda) \Rightarrow h_{n}=\lambda^{n} .
$$

Therefore the pole of $H_{+}(z)$ at $z=\lambda=\exp (2 \gamma)$ leads to exponential growth of the system function $h_{n}$ which in turn implies [eq. [25)] an exponentially increasing mean squared amplitude $x_{n}$. The growth exponent $\gamma$ is the so-called generalized Lyapunov exponent [3] related to the localization length by $\xi=\gamma^{-1}$.

The value of the Lyapunov exponent $\gamma$ defines the phase. We start from the mathematical definition that all states with $\gamma \neq 0$ belong to the insulating phase. The states with $\gamma \equiv 0$ on the other hand correspond to a metal. According to this definition the states with non-exponential localization also belong to the metallic phase, because they correspond to the value $\gamma \equiv 0$. We can consider these states as a bad metal, in contrast to a good metal, where one has truly delocalized states.

We would like to give here a summary of the results which emerge from an analysis of the pole diagram (for details see the Appendix). The function $Y_{D}(w, E)$ defined by eq. (31) is purely imaginary for $v=0, u>u_{0}$,

$$
u_{0}=2 p+|E|,
$$

and the system function $H_{+}(z)$ itself is real. The pole must be located (if present at all) exactly in this region of the parameter $w$. It can be found as a solution of an in general transcendental equation

$$
\sigma^{2} \Omega_{+}(u)=1,
$$


where

$$
\Omega_{+}(u)=\frac{1}{\sqrt{u^{2}-4}} \int_{0}^{\infty}\left[J_{0}(2 t)\right]^{D-1} \cos (E t) \sin (u t) d t .
$$

For high-dimensional systems with $D \geq 4$ the function $\Omega_{+}(u)$ has a maximum at $u=u_{0}$ and decreases monotonically for $u>u_{0}$. The system function has a pole if the disorder exceeds a critical value, $\sigma>\sigma_{0}(E)$, where

$$
\sigma_{0}(E)=\Omega_{+}\left(u_{0}\right)^{-1 / 2} .
$$

Therefore, in high-dimensional systems exponential localization takes place only if the disorder is strong enough.

For $\sigma<\sigma_{0}(E)$ the function $H_{+}(z)$ has no poles, its ROC its $|z| \geq 1$. This means that the unit circle $|z|=1$ belongs to the ROC, and the filter is stable.

We interpret solutions for this range of the disorder values, $\sigma$, also as localized, however with non-exponential localization. Non-exponential localization corresponds to a Lyapunov exponent $\gamma=0$. This simply means that the corresponding function grows slower than the exponent $\exp (2 \gamma n)$. To this class of functions do not only belong power-law functions, but also exponents with a different argument, e.g. $\exp \left(c \cdot n^{\delta}\right)$ with $\delta<1$. Here we encounter limits of applicability of the method, which come into play, however, only in physically inaccessible systems of high dimensionality. The elementary pole search can be applied only for exponentially localized states, the general case requires a detailed investigation of the filter. In its present form our theory is not capable to determine the type of non-exponential localization.

The curve for $\sigma_{0}(E)$ (Fig.1a) is the well-known mobility edge. For $\sigma>\sigma_{0}(E)$ there exists an insulating phase, whereas for $\sigma<\sigma_{0}(E)$ a metallic phase is found (bad metal). A coexistence of phases is here not possible. Thus the phase transition here has an appearance as if it were a transition of second order. This simple idea, however, is contradicted by the behaviour of the Lyapunov exponent: the transition from $\gamma \equiv 0$ to $\gamma \neq 0$ is not continuous. One can clearly see that all these mobility edges for high-dimensional systems show the same qualitative behaviour.

\section{Stability and poles: solution $H_{-}(z)$}

Now let us turn to the second branch of the solution, $H_{-}(z)$. In this case existence of the poles leads to principally different consequences. Let us assume that the corresponding value of the parameter $w$ is found and the pole $z=\lambda_{1}$ is located inside the unit circle, $\left|\lambda_{1}\right|=1 / \lambda$ with $\lambda>1$. Formally, however, from the definition $w=-\left(z^{1 / 2}+z^{-1 / 2}\right)$ the same value of $w$ can be obtained for $z=\lambda_{2}=1 / \lambda_{1}=\lambda$. The complex number $\lambda_{2}$ lies outside the region of definition of the solution, $|z| \leq 1$. In this sense the pole at $z=\lambda_{2}$ is virtual. For the inverse $Z$-transform this fact is, however, irrelevant. The ROC for a causal filter is defined by the inequality $|z|>\max \left|\lambda_{i}\right|$ or $|z|>\lambda>1$. Since the ROC and the region of definition of the solution $|z| \leq 1$ do not intersect, a physical solution is absent. Therefore, the filter $H_{-}(z)$ as a physical solution is acceptable only if either there are no poles or they lie on the unit circle. The latter case is realized for $D=2[8]$ and corresponds to so called marginal stability [18]. In the following, we consider the general case $D \geq 4$ from a unified point of view.

A pole of the first type is related to the singularity of the function eq. (34) at $w=2$ (the root, for details see the Appendix). We define the phase and amplitude via the integral (31)

$$
Y_{D}(w, E)=I_{D}(w, E) \exp \left(i \vartheta_{D}(w, E)\right) .
$$

It is not difficult to show that this pole emerges at arbitrarily small disorder for a negative phase $\vartheta_{D}(2, E)<0$. The equation $\vartheta_{D}\left(2, E_{0}\right)=0$ defines the boundary of the region $|E|>E_{0}$, where the physical solution is absent and, therefore, any disorder transforms the delocalized states into localized ones. For high-dimensional systems the delocalized states transform into states with non-exponential localization. The corresponding $E_{0}$ values are $E_{0}=3.915$ $(\mathrm{D}=4), E_{0}=4.365(\mathrm{D}=5)$ and $E_{0}=4.578(\mathrm{D}=6)$.

For the region $|E|<E_{0}$ there exists the physical solution with $\sigma<\sigma_{0}^{\prime}(E)$, where $\sigma_{0}^{\prime}(E)$ is a second threshold disorder value. The behaviour of this curve $\sigma_{0}^{\prime}(E)$ (Fig.1b) is determined by resonance phenomena. The integral in (31) consists asymptotically of a power function, $t^{(D-1) / 2}$, and a product of trigonometric functions. E.g. the function $\cos (2 t-\pi / 4)$ comes from every Bessel function. If we represent this product as a sum of monochromatic waves, then we denote the existence of a wave with zero frequency as a resonance. For $w=0$ the first non-trivial resonance lies either at $E_{c}=2(\mathrm{D}=4,6, \ldots)$ or at $E_{c}=4(\mathrm{D}=5,7, \ldots)$.

For $|E|<E_{c}$ a pole of the second type appearing at higher levels of disorder $\sigma>\sigma_{0}^{\prime}(E)$ must be considered. This type of pole emerges at purely imaginary values of the parameter $w=i v$. It corresponds to the roots of the equation

$$
\sigma^{2} \Omega_{-}(v)=1,
$$




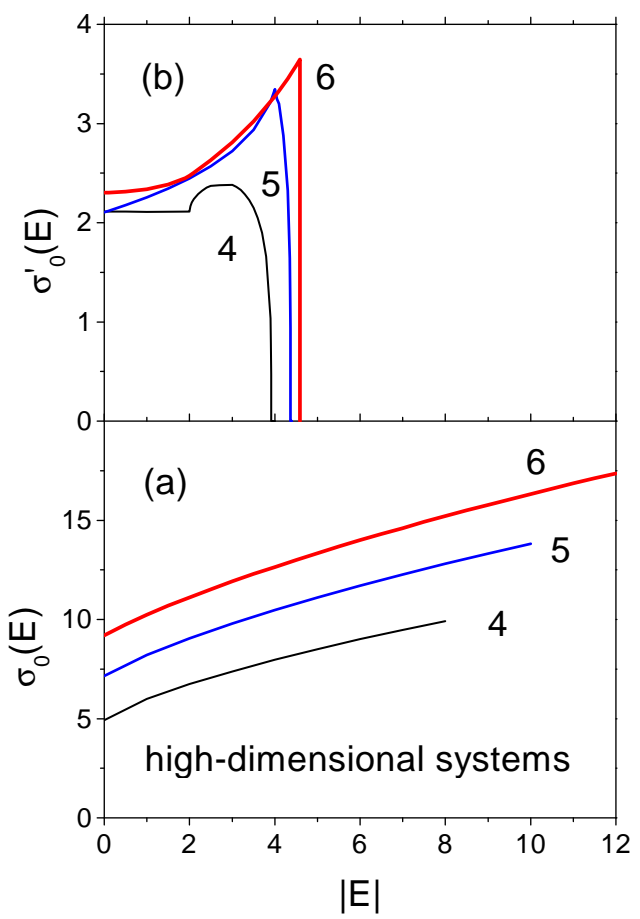

FIG. 1: Threshold disorder values: (a) $\sigma_{0}(E)$ for the transition from the non-exponential to the exponential localization and (b) $\sigma_{0}^{\prime}(E)$ for the transition from the delocalized to the (non-exponentially) localized states. The curves are enumerated with the values of $D$.

where

$$
\Omega_{-}(v)=\frac{1}{\sqrt{4+v^{2}}} \int_{0}^{\infty}\left[J_{0}(2 t)\right]^{D-1} \cos (E t) \exp (-v t) d t .
$$

A physical solution is acceptable here only if the poles lie on the unit circle, $|z|=1$ or $w \in[0,2]$ for our sector w. A marginally stable solution corresponds to the value $w=0$ (or $v=0$ in eq. (42)). The threshold disorder value is given by

$$
\sigma_{0}^{\prime}(E)=\Omega_{-}(0)^{-1 / 2} .
$$

For $\sigma<\sigma_{0}^{\prime}(E)$ the function $H_{-}(z)$ does not possess poles, here we find the region of stability of the extended states.

Spaces of dimension $D=4,5$ possess a certain sensitivity with respect to the resonance phenomena mentioned above. For $E_{c}<|E|<E_{0}$ the line of the poles exhibits a deviation from purely imaginary values of the parameter $w$ and touches the real axis at the point $u^{\prime} \in[0,2]$. This point corresponds to the condition for the phase of the integral $\vartheta_{D}\left(u^{\prime}, E\right)=0$ and also belongs to the unit circle (marginal stability). The corresponding threshold disorder value we denote again as $\sigma_{0}^{\prime}(E)$ :

$$
\sigma_{0}^{\prime}(E)=\left(\frac{\sqrt{4-u^{\prime 2}}}{I_{D}\left(u^{\prime}, E\right)}\right)^{1 / 2} .
$$

We see that the function $\sigma_{0}^{\prime}(E)$ is in general singular in the energy. In going from the energy value $E_{c}$ to the value $E_{0}$ the parameter $u^{\prime}$ increases monotonically and reaches finally the value $u^{\prime}=2$. The function $\sigma_{0}^{\prime}(E)$ goes continuously to zero for $|E| \rightarrow E_{0}$. 
For dimensions $D \geq 6$ there are no resonance phenomena. These resonances are so weak that only one equation (43) remains valid in the whole range of energies $|E|<E_{0}$. I. e. although $D_{c}=4$ is a certain critical dimension (here non-exponential localization arises), a qualitative agreement of all results is only obrtained for $D \geq 6$.

In this way it emerges from our exact analytic theory that for Anderson localization the upper critical dimension is $D_{0}=6$, i.e. the problem in a certain way shows a similarity to percolation [30] - and this may not be totally unexpected. However, we also note that as a rough estimate for the upper critical dimension the value $D_{c}=4$ may also be accepted. It corresponds to a first and important step of the qualitative saturation of the results (the possibility of existence of non-exponentially localized states), whereas a complete saturation only obtains at $\mathrm{D}=6$.

Quite generally in the range $|E|<E_{0}$ and under the condition $\sigma<\sigma_{0}^{\prime}(E)$ there exists a region of existence of stable delocalized states. Because in this range also non-localized states coexist with the other ones, this means that in this range the good metal (delocalized states) and the bad metal (non-exponentially localized states) can form a heterogeneous system, whose properties depend on the relative proportions of the subsystems; e.g. the bad metal, if the subsystem of non-exponentially localized states percolates. Fig. 1 presents results of a numerical solution of the resulting equations.

\section{LOW-DIMENSIONAL SYSTEMS}

\section{A. Analytic solution}

Independently from whether $D_{c}=4$ or $D_{0}=6$ are taken as the upper critical dimension, it is necessary to consider 3 -D systems as low dimensional ones. We approach here a widely held opinion that all states in $D=3$ should be stable against perturbations and should have a finite radius of convergence. This, however, is not correct and would only be valid if the whole field of the scaling theory of localization would be faultless.

The eq.(37) has always a solution for the physically important cases $D=2,3$. We refer to these cases as 'lowdimensional' ones. For the low-dimensional systems the integral eq. (38) can be evaluated analytically (the formulas can be found in the tables of Laplace transforms). The corresponding pole diagrams are shown graphically in the Appendix. The system function has a pole at $z=\lambda=\exp (2 \gamma)$ with $\gamma>\gamma_{0}, 2 \cosh \left(\gamma_{0}\right)=u_{0}=2 p+|E|$ and $p=D-1$. Note, however, that the above mentioned feature of the low-dimensional systems is caused by the divergence of the integral $\Omega_{+}\left(u_{0}\right)$.

If one applies the obtained equations (43) for $\sigma_{0}(E)$ to low-dimensional systems with $D=2,3$, then formally $\sigma_{0}(E) \equiv 0$. Therefore, even infinitesimal disorder leads to solutions with exponential localization. Because the curve $\sigma_{0}(E)$ forms the border between exponentially localized and non-exponentially localized states, this simply means that in this model for low-dimensional systems non-exponentially localized states are impossible (see, however, a discussion below).

For such systems the second curve $\sigma_{0}^{\prime}(E)$ takes over the role of the mobility edge. The shape of this curve (Fig.2.b) for low-dimensional systems has a certain similarity with the ones for high-dimensional systems. Here we must stress that $\sigma_{0}^{\prime}(E)$ does not represent a true mobility edge, but an upper limit for the coexistence of the two phases.

For $D=2$ in [8] it has been found analytically that $\sigma_{0}^{\prime}(E)=2\left(1-E^{2} / 4\right)^{1 / 4}$ for $E<E_{0}$ where $E_{0}=2=D$. I.e. in this case the value $E_{0}$ corresponds exactly to the midst of the band half-width $2 D=4$. For $E>E_{0}$ any disorder transforms the delocalized states into localized ones.

For $D=3$ the value $E_{0}=3=D$ corresponds again to the midst of the band half-width $2 D=6$. The resonance value mentioned above $E_{c}$ lies for $D=3$ at $E_{c}=4$. Because $E_{0}<E_{c}$, the resonance does not play a role here. The mobility edge $\sigma_{0}^{\prime}(E)$ again follows from eq. (43).

The case of the 3-D system shows an exception in the energy range $E_{0}<|E|<E_{0}^{\prime}=3.367$, where the function $\sigma_{0}^{\prime}(E)$ exhibits so-called reentrant behaviour. The proof of this requires a special investigation (see pole diagrams in the Appendix).

Note that for the 3-D system in the band center $\sigma_{0}(0)=0$, because the integral in eq. (42) diverges. Fig. 2 presents results of a numerical solution of the resulting equations. These results are compared to the analytical result of [8] for the two-dimensional system (curve 2).

\section{B. Phase diagram and logarithmic divergence}

Although the results for $D=3$ are formally exact, they require a special discussion. The mathematically correct results are physically acceptable only if they are stable against small perturbations (e.g. small changes in the model definition, fluctuations of parameters in the model). From this point of view two results have to be questioned: (i) the singularity of $\sigma_{0}^{\prime}(E)$ in the band center, $\sigma_{0}(0)=0$, and (ii) the non-existence of non-localized states, $\sigma_{0}(E) \equiv 0$. Point 


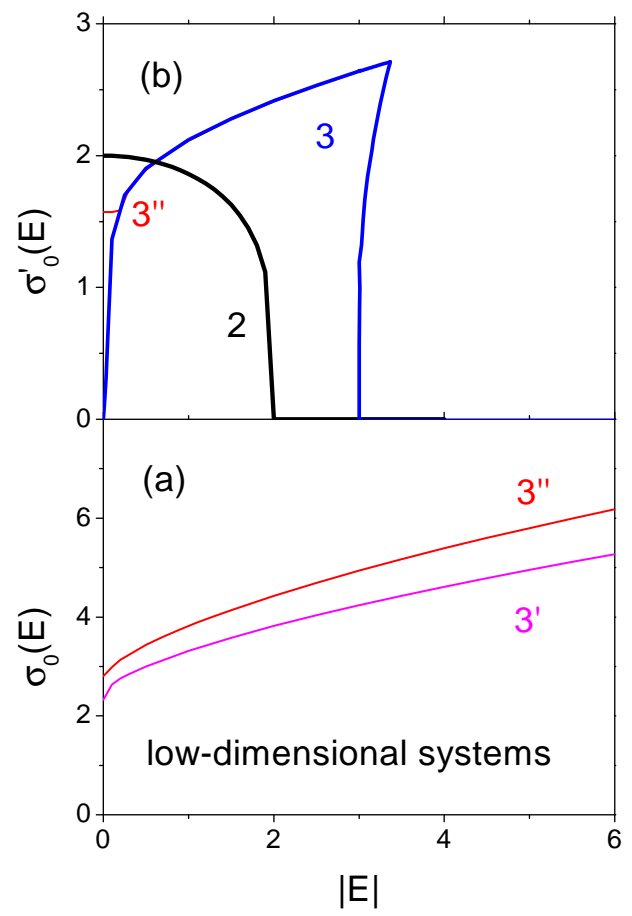

FIG. 2: Threshold disorder values: (a) $\sigma_{0}(E)$ for the transition from the non-exponential to the exponential localization and (b) $\sigma_{0}^{\prime}(E)$ for the transition from the delocalized to the localized states. The curves are enumerated with the values of $D$. The curves $3^{\prime}$ and $3^{\prime \prime}$ correspond to the model eq.45) for $\kappa=0.01$ and $\kappa=0.1$.

(i) arises mathematically as a consequence of the logarithmic divergence of the integral 42) for $v=0$ and $|E| \rightarrow 0$. Point (ii) arises again via a logarithmic divergence of the integral (38) for $u=u_{0}$. I.e. basically, the case $D=3$ is nothing else but a type of logarithmic deviation from the high-dimensional case. Under certain conditions one can find the perturbations which are capable of regularizing the mentioned logarithmic divergence, i.e. to transform them into a finite term. One could e.g. surmise that in this regularization correlated disorder [31] might play an important role. One must stress here that we are dealing only with results for tight-binding Hamiltonians with diagonal disorder. It is largely unclear, whether this property remains valid also for non-diagonal disorder.

To illustrate this let us first consider a purely mathematical problem. Is it at all possible to confirm the results (i) and (ii) either by different numerical computations or analytical approaches? The answer is - no! Any deviation from the exact solution (via numerical or analytical ways) automatically generates results of a mean-field theory. A meanfield theory has a certain qualitative agreement with the results of the exact theory, but only for high-dimensional systems. Everything looks as if any uncontrolled deviation from the exact theory has added additional dimensions to a 3-D system. As a mathematical model let us further consider a 4-D system, where, however, a coupling involving this additional dimension is exceedingly weak (parameter $\kappa \ll 1$ ). We start from a generalization of the function (20) for $D=3$ :

$$
\mathcal{E}(\mathbf{k})=E-2 \sum_{j=1}^{2} \cos \left(k_{j}\right)-2 \kappa \cos \left(k_{3}\right) .
$$

Here the term involving $\kappa$ corresponds to the hopping matrix element into the fourth dimension. Fig 2 gives the numerical results for 2 cases: $\kappa=0.01$ (curve $\left.3^{\prime}\right)$ and $\kappa=0.1$ (curve $\left.3^{\prime \prime}\right)$. One clearly sees that all results, which have nothing to do with the logarithmic divergence, e.g. the entire curve $\sigma_{0}^{\prime}(E)$ with the exception of the point $E=0$, remain extremely stable. Even the change in the parameter $E_{0}$ is of the order of $O\left(\kappa^{2}\right)$. 
The 'logarithmic' results on the other hand turn out to be completely unstable. Even the small values of the coupling (or regularization) parameter $\kappa$ produce results which are in qualitative agreement with those for high-dimensional systems. The curves $3^{\prime}$ and $3^{\prime \prime}$ look as if they were an extrapolation of the corresponding curves for $\sigma_{0}(E)$ from Fig. 17. Here the inequality $\sigma_{0}(E)>\sigma_{0}^{\prime}(E)$ also applies. Reducing the parameter $\kappa$ slowly moves the mobility edge $\sigma_{0}(E)$ downwards, because the dependence on the parameter $\kappa$ is extremely week $(\operatorname{logarithmic}), \sigma_{0}(E) \sim 1 / \ln \left(\kappa^{-1}\right)$. Only for even smaller values of the parameter $\kappa \ll 0.01$ one might perhaps see traces of the exact results, because in this case one has $\sigma_{0}(E)<\sigma_{0}^{\prime}(E)$ for $|E|<E_{0}$.

\section{CONCLUSION}

Although the formal investigation of the Anderson model of localization (tight-binding Hamiltonian with diagonal disorder) for higher spatial dimensions D might at first look very abstract, the exact analytical results supply us with clear physical consequences. The Anderson problem of localization and the percolation problem belong to the same class of critical phenomena: both have the same lower and upper critical dimensions. I.e., although the Anderson model appears to be much more complex and richer, certain fundamental results appear to be transferable. Percolation is possible for 2-D systems, this corresponds to the existence of a metal-insulator transition in disordered 2-D systems. In this sense there is no reason to believe that the existing contradictions between theory and experiment for 2-D systems point to an incompleteness of the Anderson model. On the contrary, our analytical investigation has shown that a tight-binding Hamiltonian is presumably sufficient for this purpose.

The main problem of the theory thus does not rest in the Hamiltonian, but rather in the interpretation of the results, which mainly derive from numerical work. Our analytical and exact results demonstrate the necessity of interpreting the phase transition in the framework of first order phase transition theory and this holds independently of the spatial dimension $D \geq 2$. If one, however, attempts to apply procedures which have only been developed for systems with a second order phase transition (and this is the general case), one does not necessarily obtain wrong numbers, but an incomplete or even wrong interpretation. See an example in [8], where an analytical (exact) scaling function for the 2-D system has the same form as obtained by numerical scaling. The physical interpretation is, however totally different. We hope that the necessary corrections of the numerical tools are possible to detect the first order phase transition.

The rather large value of the upper critical dimension for the Anderson localization (and the percolation) problem permits to consider 2-D and 3-D cases as low-dimensional systems. Thus a revisiting of the results is also necessary for 3-D systems. We have found that the 3-D case is nothing else but a type of logarithmic deviation from the high-dimensional case. As a consequence results a certain instability of the results, whose details are discussed in the text.

We give here a short summary of the main results.

- For the Anderson localization problem there exists an upper critical dimension $D_{0}=6$. This value is also characteristic of a related problem: percolation [30]. For $D \geq D_{0}$ all phase diagrams are qualitatively the same, only the corresponding critical values develop in a monotonic way. One can also say that this is the property of a mean-field theory, although in this case a mean-field theory does not exist as a closed theory.

- There exists also a second upper critical dimension $D_{c}=4$, which has a different meaning. The states with non-exponential localization are formed only for $D \geq D_{c}$, whereas for $D<D_{c}$ localized states are always exponentially localized. This second upper critical dimension $D_{c}$ divides the dimensions into two classes: high dimensions with $D \geq D_{c}$ and low dimensions with $D<D_{c}$.

The results for the nontrivial spatial dimensions $D>1$ can be summarized as follows.

(i) All states with energies $|E|>E_{0}$ are localized at arbitrarily weak disorder. The value of $E_{0}$ depends on $D$ and lies inside the band $E_{0}<2 D$.

(ii) For $|E|<E_{0}$ states are only localized if the disorder $\sigma$ exceeds a critical value $\sigma_{0}^{\prime}(E)$, otherwise a two-phase system is formed from an insulating and a metallic one. This differs from the traditional point of view which considers the localization transition as a continuous (second order) transition. Should the standard interpretation of this system in the framework of first-order phase transition theory be applicable (which still has to be investigated) one can expect that qualitatively it has similar properties as other heterogeneous two-phase systems (e.g. the coexistence of water and ice). Then percolation problems might be important.

(iii) Exponential localization always exists for the physically important cases $D=2,3$. Non-exponential localization occurs only for higher dimensions $D \geq D_{c}=4$. In this case for $|E|<E_{0}$ and $\sigma<\sigma_{0}^{\prime}(E)$ first the heterogeneous system appears, where the difference between the two phases may be small (this has to be investigated). For $\sigma_{0}(E)>$ 
$\sigma>\sigma_{0}^{\prime}(E)$ one finds a homogeneous system with non-exponential localization. Only for $\sigma>\sigma_{0}(E)$ a system with exponential localization appears.

(iv) $\sigma_{0}^{\prime}(E)$ is in general not an analytic function of the energy $E$. There exist certain resonances.

\section{Acknowledgments}

V.N.K. gratefully acknowledges the support of the Deutsche Forschungsgemeinschaft.

\section{APPENDIX A: POLE DIAGRAMS}

\section{Parametric representation of the pole diagram}

A filter $h_{n}$ is characterized by a pole diagram of its image $H(z)$. The principal definition eq. (24), together with the two other ones, eqs. (30), (33), supply us with the parametric w-representation of the pole diagram, eq.(34). Let us rewrite this relation into the form

$$
\frac{1}{H(z)}=1-\sigma^{2} R(w, E)
$$

where $R(w, E)$ is generally a complex function of the complex variable $w$ and energy $E$. The main idea is quite simple. The function $H(z)$ has its poles where

$$
\sigma^{2} R(w, E) \equiv 1
$$

An elementary requirement for this is the condition on the argument of the complex function

$$
\arg R(w, E)=0,
$$

because $\sigma$ is positive. For the given energy value $E$ eq. A3 defines one or more lines (pole lines) in the complex $w$ plain. As we have already discussed in the text, for reasons of symmetry it suffices to analyse only a sector $w=u+i v$ with $u \geq 0$ and $v \geq 0$. Let us analyse one of these pole lines. For each point $w$ on this line the position of the pole is determined via eq.(32). The corresponding value of the disorder $\sigma$ is found from eq.(A2):

$$
\sigma=R(w, E)^{-1 / 2} .
$$

Because for each value of $w$ on the pole line there is associated a value of $\sigma$, it is possible to indicate by an arrow next to the line in the diagram in which direction the poles move with increasing disorder.

\section{Filter $H_{+}(z)$}

Let us consider first the simplest case, the filter $H(z)=H_{+}(z)$. Here the poles are connected with the notion of the Lyapunov exponent. In the parametric representation there exists a simple relation

$$
w=2 \cosh (\gamma)
$$

thus the eqs. A4 A5 together supply a connection between $\sigma$ and $\gamma$.

For a 2-D system it is possible to analytically evaluate the corresponding function $R(w, E)[\underline{8}]$ :

$$
R(w, E)=\frac{1}{2 \sqrt{w^{2}-4}}\left[\frac{1}{\sqrt{(w+E)^{2}-4}}+\frac{1}{\sqrt{(w-E)^{2}-4}}\right]
$$

or

$$
\left[\frac{R(w, E)=\frac{1}{2 \sqrt{w^{2}-4}} \times}{\sqrt{(w+E+2)(w+E-2)}}+\frac{1}{\sqrt{(w-E+2)(w-E-2)}}\right] .
$$


We clearly see that the function (A7) possesses for the given energy several points, where it diverges. These values $w=-2-E, w=2-E, w=E-2$ and $w=E+2$ are real. They are nothing else but the resonances discussed in the text. To detect these we investigate the $w$-parametric representation of the integral eq. (31). Every Bessel function $J_{0}(2 t)$ contributes asymptotically a trigonometric function, $\cos (2 t-\pi / 4)$. In addition there exists another energy dependent trigonometric function, $\cos (E t)$. If we represent all these functions via complex exponentials, we obtain under the integral in eq.(31) asymptotically a product of the power function and a sum (with well determined coefficients) of exponents $\exp \left[i\left(w-w_{j}\right) t\right]$. These are the resonance values $w_{j}$. In the 2 -D case we have a strong resonance (the function $R\left(w_{j}, E\right)$ diverges).

It is easy to establish that the function $R(w, E)$ satisfies eq. A3 for the real value of the parameter $w$ under the condition

$$
w \geq u_{0}=\max \left\{w_{j}\right\}
$$

where for the 2-D system $u_{0}=2+|E|$. I.e. here exists a line of poles which starts from the point $u_{0}=2+|E|($ it corresponds to the value $\sigma=0$, because here $R\left(u_{0}, E\right)^{-1 / 2}=0$ ) and moves on with increasing value of the parameter $\sigma$ always along the real axis. It is also easy to find that in this case no other pole lines exist. This result can be generalized for higher dimensions. So one can establish that the value of $u_{0}$ found from eq. (36) corresponds precisely to the condition eq. (A8): we always have to deal with the same resonance.

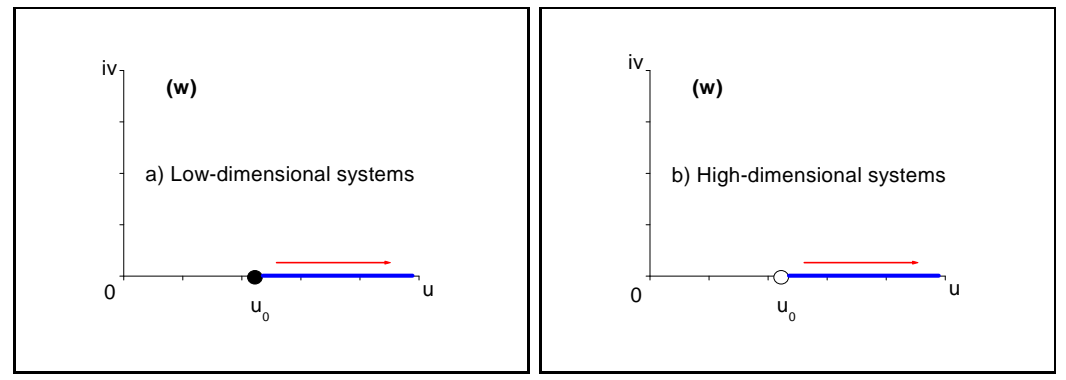

FIG. 3: Parametric representation of the pole diagram for an unstable filter: a) low-dimensional systems with $D=2,3$; b) high-dimensional systems with $D \geq 4$.

The structure of the pole diagram is nearly identical for all dimensions $D$, Fig 3 There exists only one pole line, which originates at the point $w=u_{0}$. The direction of increasing $\sigma$ is denoted by an arrow. The only difference is that for low-dimensional systems the starting point $u_{0}$ corresponds exactly to $\sigma=0$ (this is marked in the diagram with a black circle), for high-dimensional systems the value $\sigma_{0}(E)=R\left(u_{0}, E\right)^{-1 / 2}$ is finite (the point $w=u_{0}$ is marked with a white circle). In the latter case it is impossible to find for smaller values of the disorder $\sigma<\sigma_{0}(E)$ a point in the diagram, where the condition eq. (A2) is fulfilled. The filter $H_{+}(z)$ possesses no poles. The border thus found via eq.(39) has been defined as mobility edge.

Filter $H_{-}(z)$

This filter has a physical interpretation only under the condition [8] that the function $H_{-}(z)$ has either no poles or that they belong to the unit circle, $|z|=1$. In the parametric w-representation the unit circle (in the sector $w=u+i v$ with $u \geq 0, v \geq 0$ ) corresponds to an interval on the real axis $u \in[0,2]$. I.e. it is necessary first to find the single points or even lines in this interval $u \in[0,2]$, where the condition eq. A3 holds. Later on one must also find out, in which way the pole line leaves this interval.

It is easy to ascertain that one of the possible pole lines always lies on the imaginary axis $v$, and in the interval $v \geq 0$. On this line (trivial pole line) only the point $v=0$ could have a physical interpretation, because the point, $w=0$, also belongs to the unit circle. The existence of other pole lines strongly depends on the space dimension $D$ and the energy $E$.

As the simplest example let us first consider the filter for the 2-D system [8], where the corresponding function has an analytical form:

$$
R(w, E)=\frac{1}{2 \sqrt{4-w^{2}}}\left[\frac{1}{\sqrt{4-(w+E)^{2}}}+\frac{1}{\sqrt{4-(w-E)^{2}}}\right]
$$


Because the interval $u \in[0,2]$ plays an important role for the physical interpretation, we consider first the resonance values $\left\{w_{j}\right\}$, which lie precisely in this region. It is easy to calculate that for each energy value only one resonance $w=u_{0}$ exists. For energies $0 \leq|E|<2$ we have $u_{0}=2-|E|$. In the remaining energy region, $2<|E|<4$, one has $u_{0}=|E|-2$. In the 2-D system the resonances are so strong (the function $R(w, E)$ diverges at $w=u_{0}$ ) that these determine in a unique manner the beginning of the new pole lines and their direction, Fig 4

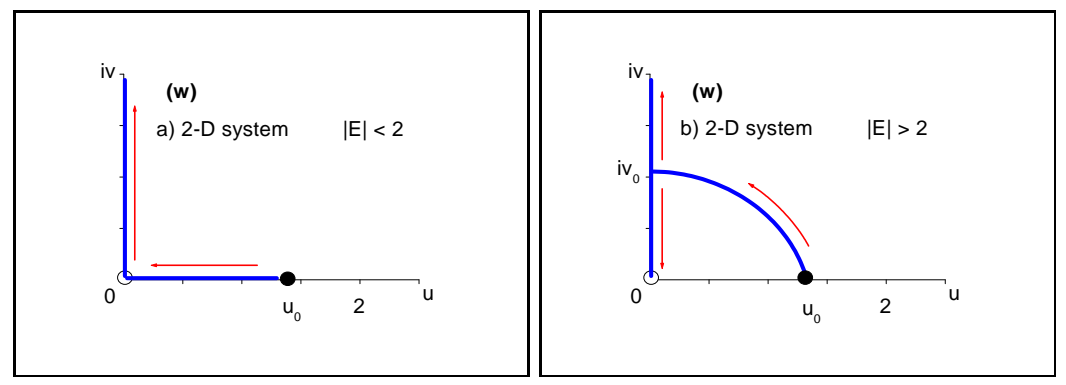

FIG. 4: Parametric representation of the pole diagram for a 2-D system: a) energy range $0 \leq|E|<2$; b) energy range $2<|E|<4$.

In the interval $0 \leq|E|<2$ the pole line starts exactly at the point $u_{0}=2-|E|$. This point corresponds to the value of the disorder $\sigma=0$ and is denoted by a black circle in the diagram. With increasing value of $\sigma$ the pole line follows exactly the real axis $u$, until it reaches the point $u=0(w=0)$. At this point the corresponding disorder $R(0, E)^{-1 / 2}$ is finite (white circle in the figure), and we have denoted this in eq. (43) as $\sigma_{0}^{\prime}(E)$. Because this line is always a unit circle (marginal stability), we have physical solutions. These exist only under the condition that $0 \leq \sigma<\sigma_{0}^{\prime}(E)$. If the disorder crosses the border $\sigma_{0}^{\prime}(E)$, the pole line leaves the point $w=0$ and follows further the trivial pole line (imaginary axis). Here, however, a physical interpretation is no longer possible and consequently there are no extended states in the range $\sigma>\sigma_{0}^{\prime}(E)$.

In the interval $2<|E|<4$ the behaviour of the pole line determines the resonance at $w=u_{0}=|E|-2$. This line again starts at the value $u_{0}$, which corresponds to the disorder $\sigma=0$ (black circle in the figure). Then this line leaves the real axis and takes its course into the complex plain until it reaches a particular point $v_{0}$ (bifurcation point) on the imaginary axis $v$. The later path makes use of branches of trivial pole lines. The directions belonging thereto are again marked with an arrow. Thus we have for $\sigma>0$ no points in the phase diagram which can be interpreted physically. I.e. an infinitesimal disorder already suffices in this energy range to destroy all extended states. One may also write that in this range $\sigma_{0}^{\prime}(E) \equiv 0$.

Formally between the 3 -D case (low-dimensional system) and the cases $D \geq 4$ (high-dimensional systems) there is a quantitative difference. For high-dimensional systems one does not find a divergence of the function $R(w, E)$ at the resonance $w=u_{0}$, but only a jump in the argument of this complex function. For the 3-D system this divergence exists, on the other hand, but in contrast to the 2-D system the resonance is very weak (logarithmic divergence). In all these cases the resonances have no direct influence: they do not generate a pole line emerging from the point $u_{0}$. There is, however, an indirect influence of the resonances, because every resonance defines the argument of the function $R(w, E)$.

In fig [5] different types of pole diagrams are presented. Fig 5 a is typical for small values of the energy. Although the resonance at $w=u_{0}$ exists (this point is marked in the figure with a black square), the weak resonance does not generate a new pole line. There remains only the trivial pole line, where only the point $w=0$ has physical significance. The value $R(0, E)^{-1 / 2}$ defines again the function $\sigma_{0}^{\prime}(E)$ already mentioned. Physical solutions exist only for $\sigma<\sigma_{0}^{\prime}(E)$ (no poles on the unit circle). Fig 5 b is typical for large values of the energy. In the parametric representation of the filter function, eq.(34), one can in addition see a point, where the function $R(w, E)$ diverges. This is the point $w=2$ (a type of energy independent resonance, which corresponds to the factor $\sqrt{4-w^{2}}$ ). One can see that this point takes over the role of the resonance at $u_{0}$. The pole line starts from the point $w=2$ (this point corresponds to the value $\sigma=0$ and is therefore again marked with a black circle). The continuation is similar to Fig 4 $\mathrm{b}$ : the line continues to the imaginary axis and reaches it at the point $v=v_{0}$. Although the points $w=u_{0}$ and $w=2$ are not identical, Fig 40 and Fig 50 are qualitatively similar, and they have the same physical interpretation. In this range of the energy an infinitesimal disorder destroys all extended states.

An indirect influence by the resonances consists in the fact that one can change in their neighbourhood the argument of the complex function $R(w, E)$ in such a way that the condition eq. A3 becomes valid at a point $w=u^{\prime}$, see Fig 5 . and Fig 5]. The point $u^{\prime}$ is not a resonance, here the function $R\left(u^{\prime}, E\right)$ remains finite (white circle in the pole diagram). 


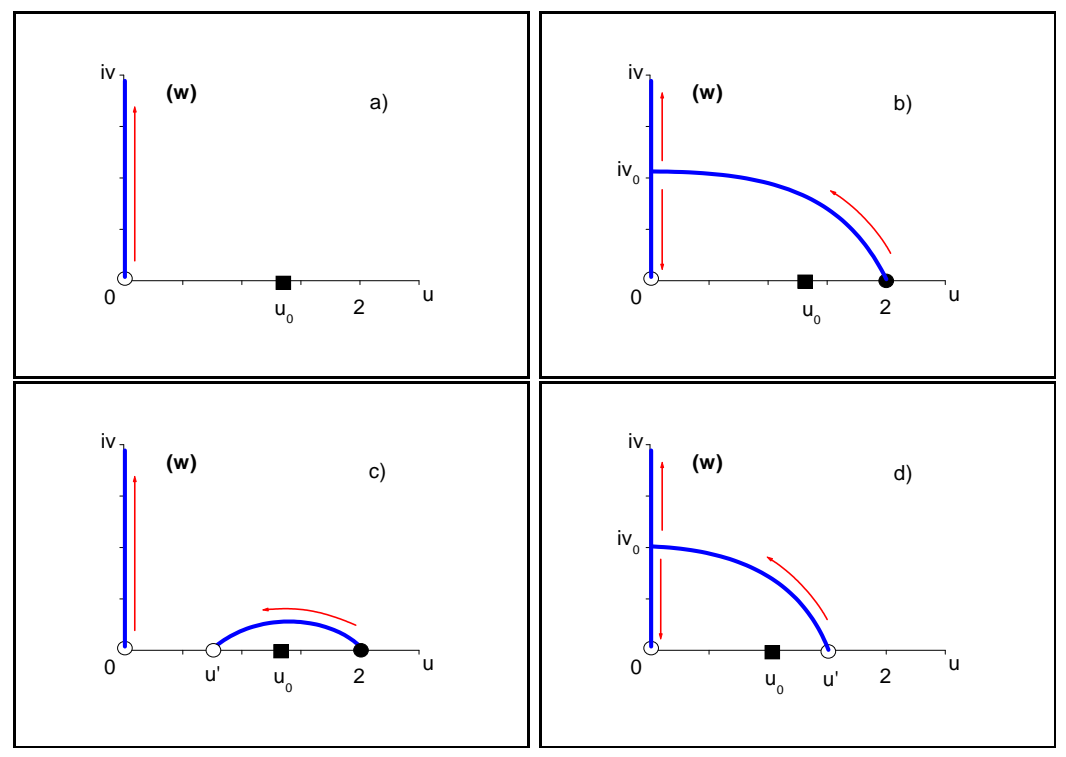

FIG. 5: Different types of pole diagrams for the systems with spatial dimensions $D \geq 3$. For details see the text.

Let us consider further on two examples. In the 3-D case one can divide the energy values into three ranges. In the range $0 \leq|E|<2$ the resonance is found at $u_{0}=|E|$, which, however, has no importance. We have the pole diagram of Fig 5 . In the range $2 \leq|E|<4$ the resonance occurs at $u_{0}=4-|E|$. Here the above mentioned point $u^{\prime}$ arises, but under the condition $|E|>E_{0}=3.00$. I.e. in the range $2 \leq|E|<E_{0}$ the pole diagram of Fig 5 remains valid. In the range $E_{0}<|E|<4$ we find a different typ of diagram, Fig 5 . The point $u^{\prime}$ corresponds to the condition $0 \leq u^{\prime}<u_{0}$. For $E \rightarrow 4$ the point $u^{\prime}$ moves towards the value $u^{\prime}=0$, and thus arises the bridge between the new pole line and the trivial pole line. After this the diagram is qualitatively the same as Fig [5b: there are no extended states. This is also valid in the range $4<|E|<6$, where $u_{0}=|E|-4$.

Figure 5. describes a complicated case which does not have an unambiguous interpretation. Formally this is the only diagram which has two pole lines. The other ones consisted always of a single pole line, although bifurcation points were also possible. For infinitesimally small disorder the resonance at $w=2$ is important, as in the diagram of Fig [5. Here with increasing disorder $\sigma$, the pole line which starts at the point $w=2$ (black circle), leaves the interval $u \in[0,2]$. The corresponding poles have no physical interpretation, which corresponds to the annihilation of extended states via infinitesimal disorder. If the disorder increases further this pole line approaches again the interval $u \in[0,2]$, and reaches it at the point $u^{\prime}$ (Fig. 囵). The corresponding value of the disorder $\sigma_{1}=R\left(u^{\prime}, E\right)^{-1 / 2}$ is finite (white circle). The physical interpretation now depends to which value of the disorder $\sigma_{2}=R(0, E)^{-1 / 2}$ corresponds the point $w=0$ (white circle) on the trivial pole line.

If $\sigma_{1}<\sigma_{2}$, then a sort of gap arises in the disorder in such a manner that in the range $\sigma_{1}<\sigma<\sigma_{2}$ no values of the parameter $w$ correspond to the pole line. I.e. the filter $H(z)$ has no poles in this range. Consequently a physical interpretation of the solution is possible here and we obtain the reappearance of extended states at finite disorder values in this special interval. This condition, $\sigma_{1}<\sigma_{2}$, is valid for 3-D systems only in a very narrow range of the energy, $E_{0}<|E|<E_{0}^{\prime}$, where $E_{0}^{\prime}=3.367$. The behaviour of the curve $\sigma_{0}^{\prime}(E)$ is shown in Fig 2 (so-called reentrant behaviour). We can clearly see that to each energy value in the range $E_{0}<|E|<E_{0}^{\prime}$ are associated three values of $\sigma_{0}^{\prime}(E)$; these are in particular $\sigma_{0}^{\prime}(E)=0$ - localization via infinitesimal disorder, $\sigma_{0}^{\prime}(E)=\sigma_{1}$ - reappearance of extended states, and $\sigma_{0}^{\prime}(E)=\sigma_{2}$ - again localization.

Outside this range, $E_{0}^{\prime}<|E|<4$, one has $\sigma_{1}>\sigma_{2}$. For this condition there are no points on the pole line which permit a physical interpretation. I.e. after this annihilation of extended states via infinitesimal disorder it is not possible for extended states to reappear.

In the 4-D case we find a different sequence of resonances. In the range $0 \leq|E|<2$ the resonance occurs at $u_{0}=2-|E|$, and the diagram Fig 5 a is valid. In the range $2<|E|<4$ the resonance is found at $u_{0}=|E|-2$. Here the point $u^{\prime}\left(u_{0}>u^{\prime} \geq 2\right)$ arises. The pole diagram corresponds to 5 t. The pole line starts from a white circle, which corresponds to the value $\sigma_{0}^{\prime}(E)>0$. Only this point in the pole diagram has a physical interpretation, and it means the existence of extended states under the condition of small values of the disorder parameter, $\sigma<\sigma_{0}^{\prime}(E)$. For $E \rightarrow 4$ the values $u^{\prime}$ and $u_{0}$ move towards $w=2$. Because always $u^{\prime}>u_{0}$, this means that the point $u^{\prime}$ reaches the value $w=2$ before the point $u_{0}$, and this occurs for $E_{0}=3.915<4$. For $E>E_{0}$ the diagram [5] is still valid, although 
the series of resonances is not yet exhausted: a resonance arises at $u_{0}=6-|E|$ for $4<|E|<6$, and another one at $u_{0}=|E|-6$ for $6<|E|<8$. Here also extended states are not possible.

\section{REFERENCES}

[1] P.W. Anderson, Phys. Rev. 109, 1492 (1958).

[2] E. Abrahams, P.W. Anderson, D.C. Licciardello, and T.V. Ramakrishnan, Phys. Rev. Lett. 42, 673 (1979).

[3] L.Molinari. J.Phys.A: Math. Gen. 25, 513 (1992).

[4] E. Abrahams, S.V. Kravchenko, M.P. Sarachik, Rev. Mod. Phys. 73, 251 (2001).

[5] S.V. Kravchenko, D. Simonian, M.P. Sarachik, W. Mason, and J.E. Furneaux, Phys. Rev. Lett. 77, 4938 (1996).

[6] S. Ilani, A. Yacoby, D. Mahalu and H. Shtrikman. Science 292, 1354 (2001)

[7] S. Ilani, A. Yacoby, D. Mahalu and H. Shtrikman, Phys. Rev. Lett. 84, 3133 (2000)

[8] V.N. Kuzovkov, W. von Niessen, V. Kashcheyevs and O. Hein, J. Phys.: Condens. Matter, 14, 13777 (2002).

[9] N.F. Mott, in Electronics and Structural Properties of Amorphous Semiconductors, edited by P.G. Le Comber and J. Mort (Academic, London, 1973), p.1.

[10] P.A. Lee and T.V. Ramakrishnan, Rev. Mod. Phys. 57, 287 (1985).

[11] P. Markoš, L.Schweitzer and M.Weyrauch, J. Phys.: Condens. Matter, 16, 1679 (2004).

[12] V.N. Kuzovkov, V Kashcheyevs, and W. von Niessen, J. Phys.: Condens. Matter, 16, 1683 (2004).

[13] L. Landau, Phys. Zs. Sowjet, 11, 26 (1937).

[14] H. Bethe, Proc. Roy. Soc., 150, 552 (1935).

[15] L. Onsager, Phys. Rev., 65, 17 (1944).

[16] V.L. Ginzburg, L.D. Landau, Zh. Eksp. Teor. Fiz., 20, 1064 (1950).

[17] J. B. Pendry, J. Phys. C: Solid State Phys., 15, 3493 (1982).

[18] T.F.Weiss. Signals and systems. Lecture notes. http://umech.mit.edu/weiss/lectures.html

[19] J.B. Pendry and E. Castano, J. Phys. C: Solid State Phys. 21, 4333 (1988).

[20] B. Kramer and A. MacKinnon, Rep. Prog. Phys. 56, 1469 (1993).

[21] H. Grussbach and M. Schreiber, Phys. Rev. B, 51, 663 (1995).

[22] T.M. Rice, Nature 389, 916 (1997).

[23] H. Kunz and B. Souillard, J.Phys. (Paris) Lett. 44, 503 (1983).

[24] I. Travěnec and P. Markoš, Phys. Rev. B, 65, 113109 (2002).

[25] S.L.A. de Queiroz, Phys. Rev. B, 66, 195113 (2002).

[26] H.E. Stanley, Introduction to Phase Transition and Critical Phenomena (Oxford Univ. Press, New York, 1971).

[27] S. Ma, Modern Theory of Critical Phenomena (Benjamin, London, 1976).

[28] V. Kuzovkov and E. Kotomin, Rep. Prog. Phys. 51, 1479 (1988).

[29] E. A. Kotomin and V. N. Kuzovkov, Modern Aspects of Diffusion-Controlled Reactions: Cooperative Phenomena in Bimolecular Processes, Vol. 34 of Comprehensive Chemical Kinetics (Elsevier, North Holland, Amsterdam, 1996).

[30] D. Stauffer and A. Aharony Introduction to Percolation Theory (Taylor and Francis, London, 1992).

[31] F.A.B.F. de Moura and M.L. Lyra, Phys.Rev. Lett., 81, 3735 (1998). 